\title{
SwissDRG: Eine sorgfältig ausgearbeitete Tarif- struktur ist der Garant für Stabilität bei der bevorstehenden Reform der Spitalfinanzierung
}

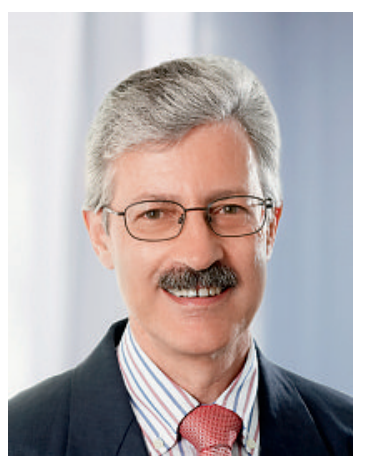

Wir alle haben wohl schon zu einem Assistenzarzt gesagt, dass jeder Patient mit seiner Krankheit, seinem Erbgut, seiner Sensibilität und seinem sozialen Umfeld ein besonderer Fall ist. Im Spitalalltag bestätigt sich dies laufend: Trotz standardisierter Verfahren ist eine individuell abgestimmte Patientenversorgung auch weiterhin erforderlich. Wie ist vor diesem Hintergrund eine umfassende Objektivierung der gesamten medizinischen Versorgung vorstellbar? Auch wenn die benötigten Daten permanent und intensiv erhoben werden, wird eine solche Objektivierung nie vollumfänglich möglich sein, weder zu statistischen noch zu tarifären Zwecken. Es gilt somit, einen Mittelweg zu finden zwischen Genauigkeit und Informationsverzerrungen sowie zwischen administrativer Freiheit und einer endlosen Datenerfassung. Mit der Konfiguration von zahlreichen Fallpauschalen und der Integration der von den Fachgesellschaften

\section{Die Tarifstruktur garantiert uns, dass der Umfang unserer Leistungen belegt und eine angemessene Vergütung begründet werden kann.}

verlangten Differenzierungen ist die Tarifstruktur SwissDRG in Bezug auf diesen Ausgleich eine gute Lösung.

Patienten mit komplexen Erkrankungen oder einer Vielzahl von Komorbiditäten benötigen während ihres ganzen Spitalaufenthalts ein vermehrtes Engagement des gesamten medizinischen Personals. Mit dem Einbezug dieser komplexen Verfahren in die Klassifikation CHOP 2011 können diese Details nun berücksichtigt werden. Deren Kumulation entspricht einem echten Mehrwert für den Patienten, aber auch einer Belastung für das Personal. Damit die Kodierer diese Leistungen richtig und problemlos erfassen können, hat die FMH Dokumentationsmodelle erarbeitet, die von Petra Ingenpass in dieser Ausgabe der SÄZ auf Seite 357 vorgestellt werden.

In einem Artikel von Beatrix Meyer auf Seite 353 geht es um eine geplante Studie zur Verlagerung von Leistungen zwischen dem stationären und dem ambulanten Bereich, die wir in Zusammenarbeit mit H+ lancieren. Die Verlagerung von Leistungen in den ambulanten Bereich ist seit manchen Jahren zu beobachten. Sie wird von den Patienten gewünscht und ist wirtschaftlich vernünftig. Dieser Trend könnte sich noch weiter verstärken. Für die Ärzteschaft ist es wichtig, diese Entwicklung durch die Prüfung der Beweggründe zu objektivieren.

Die nächste Reform der Spitalfinanzierung ist eine Art Grossbaustelle mit mehreren Unbekannten. Die Finanzierung der Weiterbildung der Ärztinnen und Ärzte ist auf

\section{Die Ärzteschaft spielt eine sehr wichtige} Rolle, indem sie regelmässig Vorschläge ihrer Fachgesellschaften unterbreitet.

nationaler Ebene immer noch nicht klar geregelt. Die Übernahme der Investitionen ist in ihrer Höhe umstritten, die Art und das Ausmass des Datenaustausches sind weiterhin ein Stein des Anstosses. Der Preisüberwacher gibt verblüffende Erklärungen ab, die einen künftigen Versuch zu einer rücksichtslosen Beschneidung der Spitalvergütungen vermuten lassen. Angesichts all dieser Ungewissheiten ist eine solide Tarifstruktur, die seit mehreren Jahren von allen Partnern der SwissDRG AG erarbeitet wird, eine gute Sache. Mit ihren Datenerfassungen garantiert sie uns, dass der Umfang unserer Leistungen belegt und eine angemessene Vergütung begründet werden kann.

Die Version 1.0 von SwissDRG soll ab dem 1. Januar 2012 einsatzbereit sein. Sie wird noch nicht perfekt sein, doch ein wesentlich besseres Instrument darstellen als die gegenwärtig verwendeten DRG-Systeme. Mit jährlichen Anpassungen wird man sie kontinuierlich verbessern. Diesbezüglich spielt die Ärzteschaft eine sehr wichtige Rolle, indem sie regelmässig Vorschläge ihrer Fachgesellschaften unterbreitet. Denn nur die Ärztinnen und Ärzte begleiten die Patienten während der gesamten Versorgungskette. Deshalb können sie die erbrachten Leistungen am besten quantifizieren. Das menschliche Engagement dagegen, das für alle Patienten erbracht wird, lässt sich nur schwer erfassen. Dieser wichtige und zentrale Faktor unseres Berufs wird sich den wirtschaftlichen und statistischen Verfahren immer entziehen. Er ist jedoch die Garantie dafür, dass wir unsere therapeutische Freiheit beibehalten können, unabhängig von tarifären, strukturellen und politischen Entwicklungen. Aus allen diesen Gründen werden wir diesem Faktor immer eine hohe Bedeutung beimessen.

Dr. med. Pierre-François Cuénoud, Mitglied des Zentralvorstands der FMH, Verantwortlicher Ressort SwissDRG 